Available online on 15.05.2020 at http://jddtonline.info
Open Access to Pharmaceutical and Medical Research
unrestricted non-commercial use, provided the original work is properly cited

Open@Access

Review Article

\title{
Self-Emulsifying Formulations: A Pharmaceutical Review
}

\section{Agubata Chukwuma}

Department of Pharmaceutical Technology and Industrial Pharmacy, University of Nigeria Nsukka

\begin{abstract}
The oral route of drug delivery is commonly utilized for administration of medicines and is particularly preferred for the treatment of many chronic diseases which require continuous ingestion over a reasonably prolonged period of time. However the oral delivery of lipophilic drugs presents a major obstacle because of their low aqueous solubility. The aqueous solubility of a drug is a crucial determinant of its dissolution rate, absorption and bioavailability. Drugs with relatively high intrinsic lipophilicity can be dissolved in appropriate mixtures of oils/lipids, surfactants, cosolvents which can rapidly form oil-in-water $(\mathrm{o} / \mathrm{w})$ fine emulsions when dispersed in aqueous phase under mild agitation or mixing. These isotropic self-emulsifying formulations or self-emulsifying drug delivery systems are effective for delivery of poorly soluble, lipophilic drugs by dispersing the drugs within fine oil droplets in emulsions and this solubilization of drugs can then improve its absorption, bioavailability and therapeutic efficacy. The present paper reviews the concept, design, formulation, characterization and applications of selfemulsifying formulations.
\end{abstract}

Keywords: Self-Emulsifying Formulations, lipophilicity, emulsions

Article Info: Received 12 March 2020; Review Completed 16 April 2020; Accepted 26 April 2020; Available online 15 May 2020

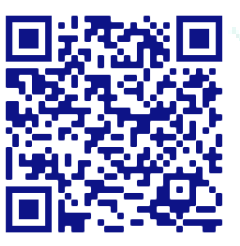

Cite this article as:

Agubata C, Self-Emulsifying Formulations: A Pharmaceutical Review, Journal of Drug Delivery and Therapeutics. 2020; 10(3):231-240 http://dx.doi.org/10.22270/jddt.v10i3.3981

*Address for Correspondence:

Agubata Chukwuma, Department of Pharmaceutical Technology and Industrial Pharmacy, University of Nigeria Nsukka

\section{Introduction}

Self-emulsifying formulations are blends of oil, surfactant, co-solvent and solubilized drug that appear as isotropic dispersions 1. These unique formulations can rapidly transform to oil-in-water (o/w) emulsions with fine droplets when dispersed in aqueous medium under mild agitation. The described preparations are known as self-emulsifying drug delivery systems (SEDDS). Oil-soluble active compounds can be dissolved or dispersed in the oily phase and distributed across the biological systems within fine oil droplets carried in aqueous medium. SEDDS are commonly referred to as self-microemulsifying drug delivery systems if they form microemulsion with nano range droplet size. The rapid emulsification of these formulations in the gastrointestinal tract can facilitate improved oral bioavailability and a reproducible plasma concentration. The self-emulsifying capacity of the formulation augments the natural emulsifying activities of the GIT bio-structure. The droplet size of the emulsion would affect the extent and rate of absorption of the orally administered drug with smaller sizes usually improving absorption, bioavailability and efficacy of bioactive compounds. A drug substance would require high lipophilic properties for it to qualify as candidate to be delivered as SEDDS since the active ingredient should be dissolved in a relatively small amount of oil. This condition is necessary so that the volume of oil required to solubilize the required total dose of the medicament would not be unnecessarily high. High chemical stability of the dissolved drug in oil phase is highly desired for the lipid formulations. The functional groups in the drug which may be vital for bioactivity should not chemically interact with groups in the oil phase which may cause reduced activity, loss of activity or emergence of unwanted toxicity.

Self-emulsifying drug delivery systems are homogenous mixtures of natural or synthetic oils, surfactants and cosolvents/cosurfactants 2 . These systems are able to selfemulsify rapidly in gastro-intestinal fluids to form fine oil/water emulsions under the gentle agitation or mixing that occurs in the gastro-intestinal tract due to peristaltic movements.

\section{Lipids}

Lipids are a group of naturally occurring substances that include fats, waxes, monoglycerides, diglycerides, triglycerides, phospholipids and other lipidic molecules. The main biological functions of lipids include storing energy, providing structural components of cell membranes and signaling 3,4. Lipids are being effectively applied in pharmacy, cosmetics, food, nanotechnology, et c. Solubilization of the drug in the oily phase and access to the lymphatic circulation depend on the type and amount of oil applied for formulation since different oils have different fatty acid constituents which are also present in different 
combinations. Digestive lipids such as tri- and diglycerides, fatty acids, cholesterol, phospholipids, and other lipids that are synthesized lead to improvement in bioavailability of the drug whereas non-digestible lipids cause reduced bioavailability due to inhibitions in absorption because there may be retention of some quantities of administered drug in the preparations. Although edible oils based on natural origin are promising, they may not be useful because they usually do not have sufficient capacity to solubilize large amount of drugs with high lipophilic properties and selfemulsification can be problematic in these cases because of the presence of large molecular volume ${ }^{5}$. The polarity of the droplets is governed by the Hydrophile-Lipophile Balance (HLB), the chain length and degree of unsaturation of the constituent fatty acid, the molecular weight of the hydrophilic section and the concentration of the emulsifier. Actually, the polarity portrays the affinity of the drug for oil and/or water, and the type of forces formed between the interacting components. A high polarity of the dispersed droplets will promote a rapid rate of release of the drug into the continuous aqueous phase. Lipids can be grouped into homolipids, heterolipids and complex lipids.

\section{Homolipids}

Homolipids are esters of fatty acids with different alcohols. The focal lipids are long chain and medium chain fatty acids linked to a glycerol molecule, and these lipids are known as triacylglycerols. The long-chain fatty acids ranging from C14 to 24 are widely found in common fat while the medium chain fatty acids ranging between $\mathrm{C} 6$ and 12 are typical constituents of coconut oil or palm kernel oil. Triacylglycerols or triglycerides have different physical and chemical properties which depend on the length of the carbon chain, the number and position of double bonds, and spatial arrangement. Most natural fatty acids occur as cisconfiguration, although trans-fatty acids are known to exist also in seed oils from different plant families (Ranunculaceae, Lamiaceae or Asteraceae), milk, meat and some partially modified dietary fats (margarine) 6 . Structured lipids are triacylglycerols that contain mixtures of any of short-chain or medium-chain fatty acids or both and long-chain fatty acids, mostly esterified on the same glycerol molecule 7. Homolipids include glycerides (fats, oils), cerides (waxes), and sterides. Diacylglycerols are found in vegetable oils in low amounts. They are formed as intermediate products in biosynthesis of triacylglycerols, as well as by acidic and enzymatic hydrolysis during oil extraction, refining process and storage.

Chemically, fats are triglycerides, triesters of glycerol and any of several fatty acids. Fats can present as solid or liquid at room temperature depending on their structure and composition. 'Oil' is usually used to refer to fats that are liquids at room temperature while 'fats' is usually used to refer to fats that are solid at normal room temperature. Fats and oils are forms of lipids. Fats can be saturated or unsaturated. Examples of fats include lard, tallow, butter, goat fat et c. Natural waxes are usually esters of fatty acids and long chain alcohols. Chemically, a wax is a type of lipid that may contain a wide variety of long chain alkanes, esters, polyesters and hydroxy esters of long-chain primary alcohols and fatty acids. Waxes are not glycerides, they lack triglycerides which are esters of glycerol and three fatty acids and this distinguishes them from fats. Fats (and Oils) are triglycerides or triacylglycerols and consist of glycerol combined with three fatty acid molecules. In addition, waxes are esters of an alcohol (except glycerol) and a single long chain acid. Cetyl palmitate is a typical wax. There are animal and plant waxes. Examples of animal wax are beeswax, earwax, shellac wax, spermaceti, et c, while plant wax includes carnauba wax, candelila wax, et c. However, 'fat' is seen as a general term which encompasses all these groups.

Modified and hydrolyzed oil of vegetable origin usually have good emulsification properties and are biocompatible. Triglycerides with long and medium chain lengths at different degrees of saturation are used in the formulation of self-microemulsifying drug delivery systems.

\section{Heterolipids}

Heterolipids include phospholipids. Phospholipids can be obtained from different biological sources. However, sources of heterolipids are largely limited to vegetable oil seeds such as soyabeans, for pharmaceutical and some nutriceutical use while the egg yolk and milk have gained importance as reliable sources of phospholipids 8 . Phospholipids are usually of the phosphoglyceride type, although phosphingolipids are also available. Phosphoglycerides are also commonly called lecithin. They are typically phosphatidylcholines.

\section{Complex lipids}

Complex lipids are usually used to describe lipoproteins. Lipoproteins are spherical lipid-protein complexes that are responsible for the transport of cholesterol and other lipids within the biological system. Based on structural examination, lipoprotein comprises an apolar core containing cholesterol esters or triacylglycerols, covered by a distinct monolayer of phospholipid in which cholesterol and one or more specific apoproteins are embedded. Basically, lipoproteins transport lipid molecules in water. These heterogenous complex aggregates of lipid and protein are abundant in biological systems and natural lipoproteins include chylomicron, very low density lipoprotein (VLDL), low density lipoprotein (LDL) and high density lipoprotein (HDL). The different existing lipoproteins vary in size, density, lipid and apoprotein composition ${ }^{9}$.

Self-microemulsifying drug delivery systems are homogenous mixtures of natural or synthetic oils, surfactants, or alternatively, one or more hydrophilic solvents and co-solvents/ co-surfactants, which upon mild agitation following dilution in aqueous media form fine oilin-water $(\mathrm{o} / \mathrm{w})$ microemulsions. The droplet sizes are usually very small allowing for transparency.

\section{Mechanism of self-emulsification and parameters}

In emulsification process, the associated free energy $(\Delta G)$ is given by the equation 1 :

$$
\Delta \mathrm{G}=\sum \mathrm{N}_{\mathrm{i}} \pi \mathrm{r}_{\mathrm{i}}^{2} \sigma-\mathrm{-}
$$

"N" is number of droplets with radius " $r$ " and " $\sigma$ " denotes interfacial energy.

Self-emulsification occurs when the change in entropy that promotes dispersion is higher than the energy needed to elevate the surface area of the test dispersion 10 . The ease with which the degree of disorder or randomness of a system can change would determine the ease of selfemulsification. The energy gradient is a key element of the process. The free energy of the conventional emulsion is directly related to the energy required to create a fresh surface between the oil and water phases and is described by the equation above. The two phases of emulsion ultimately separate with time to reduce the interfacial area and the emulsion is stabilized by emulsifying agents, which form emulsion droplets, reduce the interfacial energy and provide a barrier to prevent coalescence ${ }^{11}$. A method has been developed for quantitatively assessing the ease of emulsification by monitoring the turbidity of the oil- 
surfactant system in a water stream. The conclusion was that the emulsification process may be associated with the ease with which water penetrates the oil-water interface, with formation of liquid crystalline phase resulting in swelling at the interface, thereby causing a greater ease of emulsification 12. The thermotropic stability of selfmicroemulsifying formulations (SMEFs) and their high drug loading capacities make them potential delivery systems for low aqueous soluble drugs 13 .

The process of self-emulsification proceeds through formation of liquid crystals (LC) and gel phases. The physico-chemical and mechanistic process of drug release from SEDDS is highly dependent on LC (liquid crystals) formed at the interface, since it is most likely to affect the angle of curvature of the droplet formed and the inhibitions to drug accumulation in aqueous media 14 .

Microemulsion properties such as phase behaviour, structure of the system, temperature-dependent stability, and solubilisation capacity, depend on some characteristics of the formed interfacial films. The interfacial tension, spontaneous curvature and film rigidity are properties of the interfacial films that can be used to design effective and stable microemulsions. Other variables that are external (such as temperature) and additives (such as salt) affect multiple properties simultaneously which suggest associative capabilities. Increase in the salt concentration in an emulsion system containing a surfactant, changes the interfacial tension, adjusts the spontaneous curvature towards a favoured direction and modifies the surface-active agent's adsorption affinity and phase attraction.

The oil-water interfacial tension $\left(\gamma_{\mathrm{ow}}\right)$ is the work needed to increase the area of an interface by unit amount. Lowering $\gamma_{\text {ow }}$ to a value that favours stable emulsion formation can be achieved using surfactant-co-surfactant mixture. The inclusion of co-surfactant creates a synergistic effect that augments the benefits of surfactants. Solubilization capacities of the surfactant system are improved when such binary systems are applied. It has been discovered that temperature, pressure, chain length of oil or fatty acid components, and additives such as salt and alcohol, affect the water-oil interfacial tension in the presence of ionic or non-ionic surfactants. These factors affect the stability of emulsions and SEDDS.

When a comparable amount of water and oil is mixed in the presence of surfactant, the film curvature is known as the spontaneous curvature $\mathrm{H}$. (also known as the natural or the preferred curvature). With the weights or volumes of the oil and water approximately equal, it is believed that a spontaneous action occurs with little or no favour for the emergence of one rather than the other. In this situation, there is no constraint on the emulsion to align into curvature of any particular value, or type, so the surfactant film is free to adopt the lowest free energy state. This freedom of orientation changes with changes in relative composition or interferences. The curvature is not spontaneous if the amount of one component (either oil or water) is very small when compared with the other component. This huge difference between the two components forces or favours the curvature to take a set of values or orientations. The absolute mean value of any curvature can be calculated using some geometric models.

For easier understanding one can denote positive curvature as concave towards oil which then favours o/w emulsion and apply negative for a curvature concave towards water for water-in-oil emulsion.
The spontaneous curvature depends on the type and structure of the microemulsion components and on the prevailing conditions of the system. In a case of water-oilsurfactant system where $\mathrm{H}^{\circ}=0$ (i.e. planar interface) where the surfactant is ionic, it has been observed that salinity and temperature affect $\mathrm{H}^{\circ}$ in different, usually opposite, ways. Increment in the saline content shields the electrostatic repulsion between head groups, and this would cause reduction in the effective area per surfactant head group. This 'charge shielding' allows the head groups to consolidate. Consequently, $\mathrm{H}_{\circ}$ decreases, appear negative and hence curved toward water for a water-in-oil emulsion. Temperature increment has a significant effect of increasing dissociation of counter-ion, which promotes electrostatic repulsion between head groups, and consequently increases $\mathrm{H}^{\circ}$. Spontaneous curvature expresses the sign and magnitude of the preferred curvature of a single surfactant monolayer with smaller microemulsion droplets being more curved than larger ones. The expressions of salt and temperature effects are different for non-ionic surfactants. This is because their mode of interference on the functionality of surfactant and interfacial environment differs. Salt has very little or no significant effect on $\mathrm{H}$ - for non-ionic surfactant systems, whereas temperature has huge effect on non-ionic agents when compared with ionic surfactants. The reason for this pronounced temperature effect is that the solubility of nonionic surfactants in water or oil depends crucially on temperature. In the case of certain non-ionic ethoxylated surfactants and when spontaneous curvature does not favour any particular direction, increasing the temperature causes the surfactant to be more soluble in oil which favours orientation to form a negative (w/o) curvature. Increase in temperature causes a transition from $\mathrm{o} / \mathrm{w}$ to bicontinuous structures to w/o microemulsions. Also, amount of oil that is solubilized in the emulsion starts increasing as the temperature increases. For this category of surfactants, increments in temperature by some degrees are enough to modify the curvature from highly positive to highly negative leading to water-in-oil emulsion. Apart from temperature and salinity or electrolyte concentration, other factors that can affect $\mathrm{H}^{\circ}$ include surfactant chemical structure, use of mixed surfactants, surfactant chemical bonds, type and valency of counter ions, chemical structure and chain length of the lipid, and solubilisation of applied adjuncts in the system. Ho depends on the chemical structure of the surfaceactive molecules and it increases with increase in HLB value to favour oil-in-water microemulsion and self-emulsification. The Ho decreases as the tail length is increased for a given head group and this corresponds to rise in lipophilicity.

Surfactant film rigidity also greatly determines microemulsion properties. Helfrich (1973), established a film bending energy model and presented two elastic moduli to describe film rigidity ${ }^{15}$. These are the mean bending elastic modulus (describing rigidity) which represents the energy required to bend a unit surface area by unit value and secondly the Gaussian (or saddle-splay) modulus, which is a term used to describe film topology.

A number of attempts for predicting the likely microemulsion type that result from a mixture of oil, water and emulsifier(s) have been made. Emulsion types can be predicted using a rule (now known as Bancroft's rule), which states that water-soluble emulsifiers tend to form oil-inwater emulsions and oil-soluble emulsifiers tend to form water-in-oil emulsions ${ }^{16}$. However, this is very qualitative, and therefore, it is necessary to look at the quantitative angle. Free surfactants are always present in the continuous phase which allows for stable droplet formation in microemulsions. This also allows self-emulsification in 
SEDDS. There is an entropy change for the self-assembling of free surfactant to the interface of a single oil or water droplet for $\mathrm{o} / \mathrm{w}$ or $\mathrm{w} / \mathrm{o}$ emulsions, respectively.

The packing of molecules at the interface of oil and water, or in an aggregate, depends on the geometric characteristics of the surfactant, and these include effective area per surfactant head group $a_{h}$, the volume of the hydrophobic tail $v$, and chain length $l_{c}{ }^{17}$. These properties are vital in predicting the behavior of surfactants under different conditions. This is because the dynamics of surfactant selfassembly is modulated by these characteristics. The hydrophobic volume $v$ and tail length $l_{c}$ are determined by the steric inhibitions of repulsive interaction between surfactant tails and also determined by oil penetration. These interactive properties can be used to predict aggregate structures by utilizing the 'packing parameter' (v/ahlc). The usefulness of the packing parameter lies in its application to estimate the shape of surfactant aggregates. Surfactants with charged head groups tend to possess large head group area which supports formation of spherical micelles while those with smaller head group area arranges into cylindrical (rod-like) shapes.

One of the benefits of using combinations of surfactants is the opportunity to formulate microemulsions that are temperature-insensitive. Mixing ionic and non-ionic surfactants types can yield temperature-insensitive microemulsions or different products with varied levels of sensitivity because the dependence of the monolayer curvature on temperature is opposite for ionic and non-ionic surfactants.

The Gibbs adsorption isotherm for multicomponent system relates the changes in quantities of a component in direct contact with a surface with changes observed in the surface tension of the system. These changes in interfacial tension causes a corresponding change in surface energy and this can be applied in emulsion systems ${ }^{18}$. For a two-component system, the Gibbs adsorption equation can be expressed to show surface excesses using Equation 2:

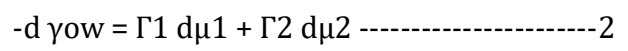

Where yow represent the surface tension, $Г \mathbf{1}$ and $Г 2$ signifies surface excesses of components 1 and 2 , and $\mu \mathbf{1}$ and $\mu 2$ are the chemical potentials of components 1 and 2 . Equation 2 relates interfacial tension to interfacial composition (or surface /interfacial excess) and chemical potential of mixture components. This relationship can be used to show the value of using both surfactant and cosurfactant or cosolvent in the formulation of self-emulsifying formulations.

\section{Advantages and limitations of SEDDS}

Some active pharmaceutical ingredients have been formulated as SEDDS and studied extensively. Some of the potential advantages of SEDDS 19 include:

1. Improved oral bioavailability which allows for dose reduction

2. Consistency and reproducibility of drug absorption profiles

3. Selective drug targeting toward a definite absorption channel in the G1 tract

4. Drug protection or 'shielding' from the hostile environment in the gut or environment

5. Controlled drug delivery

6. Presentation as liquid or solid dosage forms.
7. Reduced variability

8. High drug payloads especially when using lipids with high drug solubility

\section{Mechanism of enhanced bioavailability}

Mechanisms suggested for the reported enhanced drug absorption and bioavailability of many drugs from these selfemulsifying systems include;

- Increase in membrane fluidity that facilitates transcellular absorption,

- Opening tight junction in the biological system to allow paracellular transport,

- Inhibition of P-glycoprotein for increase in intracellular accumulation of drugs and residence time in the GIT

- Large interfacial surface area due to fine dispersed droplet size of the formulation promotes rapid release of drug substances 20 .

- Stimulating bio-production of lipoprotein and chylomicron by lipids and these products improve drug absorption and biodistribution

Certain lipids and surfactants may magnify the activity of intestinal efflux transporters as indicated by the Pglycoprotein efflux pump and may also decrease the level of enterocyte-based metabolism 21. The formation of lipoproteins promotes lymphatic transport of highly lipophilic drugs and increases the bioavailability through reduction in first pass metabolism 22. Lipids (eg triglycerides) affect the oral bioavailability of drugs by improving the dissolution rate and solubility in the intestinal fluid, protecting the active compound from chemical and enzymatic degradation and through the formation of lipoproteins promoting lymphatic transport of highly lipophilic drugs 23. Administration of lipophilic drugs using lipids as carriers may enhance drug absorption into the portal blood compared with those lacking lipids ${ }^{24}$. Many drugs degrade in the physiological system through enzymatic or hydrolytic cleavages under acidic $\mathrm{pH}$ of stomach. Such drugs when presented in form of SEDDS can be protected against these degradative processes. The use of SEDDS as vehicles for bioactive drugs provides a barrier against degradation factors.

A major limitation of SEDDS is the requirement for high concentration of surfactants which might cause some side effects such as irritation. Also the drug might be trapped in the micelles of the surfactant and this may negatively affect its availability for activity.

\section{Excipient selection in SEDDS}

Optimized pharmaceutical excipient combinations generate efficient self-emulsifying systems 25 . The mixture of oil, water and surfactants is able to form a wide range of structures and phases depending upon the proportions of each of these components. Generally regarded as safe (GRAS) excipients are usually employed in the preparations of SEDDS. Therefore a careful selection of components is usually required before SEDDS formulation.

\section{Oil/ Lipid phase}

This is an important component of any SEDDS. The main criterion for selecting the oil phase is that the drug should have a high solubility in it. Identifying the suitable oil having the maximum solubilizing capacity for the drug under study is very important to achieve optimum drug loading ${ }^{26}$. This will minimize the volume of the formulation necessary to 
deliver the therapeutic dose of the drug especially if it needs to be filled into capsules. Some natural oils can be processed to remove impurities and/or various fractions isolated and used to prepare lipid-based products. Naturally occurring oils and fats are made up of triglycerides consisting of fatty acids of varying chain lengths and degrees of unsaturation. The melting points of these lipids and their susceptibility to oxidation increases as the degree of unsaturation increases. Triglycerides are classified as short ( $<5$ carbons), medium (6-12 carbons) or long chain (>12 carbons). Long chain and medium chain triglyceride oils with different degrees of saturation are commonly used. These triglycerides may be synthetically hydrogenated to decrease the degree of unsaturation, thereby conferring resistance to oxidative degradation. Structured lipids are triacylglycerols that contain mixtures of either short chain or medium chain fatty acids or both, and long chain fatty acids, preferably esterified on the same glycerol molecule ${ }^{7}$. Highly lipophilic drug $(\log P$ $>5$ ) with high solubility in triglycerides $(>50 \mathrm{mg} / \mathrm{mL})$ can undergo lymphatic transport when co-administered with esters of unsaturated long chain fatty acids, thereby improving the bioavailability of the drug 27

Digestive lipids including triglycerides, diglycerides, fatty acids, phospholipids, cholesterol and other synthetic derivatives are suitable oils for development of delivery systems for lipophilic compounds 28 . Oils with medium hydrocarbon chain length and oils with short chains are easier to emulsify than their long chain triglyceride counterparts 29. Many combinations of lipids and their digestion products and surfactants have been shown to have permeability or absorption enhancing qualities ${ }^{30}$. It is vital to always consider the physical aging and oxidation of the oils. The choice of lipids to be used then usually depends on consideration of a whole lot of factors which are related to functionality, safety and stability.

\section{Surfactants}

The most commonly accepted surfactants for SEDDS are non-ionic surfactants possessing relatively high hydrophilelipophile-balance (HLB) values. The non-ionic surfactants are highly hydrophilic based on the hydrophilicity of the head. The hydrophilicity of the surfactants facilitates the spontaneous formation of oil-in-water emulsion globules and rapid dispersion of the preparation in aqueous media. The flexibility of the surfactant film is necessary and enables the existence of several structures including droplet-like shapes, aggregates, bicontinuous structures and multiple phase behaviours 31 . The surfactant concentration ranges between $30 \% \mathrm{w} / \mathrm{w}$ and $60 \% \mathrm{w} / \mathrm{w}$ in order to form stable SEDDS ${ }^{1}$. At higher surfactant concentrations (approximately $>50-60 \% \mathrm{w} / \mathrm{w}$ ), the progress of emulsification may be affected by the formation of viscous liquid crystalline gels at the oil/water interface. The interfaces of microemulsions are stabilized by carefully selected combinations of surfactant and/or co-surfactant based on appropriate design space to achieve quality by design (QbD). These combined agents are expected to complement each other. The addition of cosolvents such as short chain alcohols imparts flexibility to the interface that assists the free movement of the hydrophobic tails of surfactant at interface and this would consequently affect dynamic behavior of microemulsions 32 . The ingredient mixtures with higher surfactant and cosurfactant / oil ratios resulted in the formation of selfmicroemulsifying formulation 33 . Some naturally occurring polar lipids have emulsifying capacity.

There is an increasing interest in the use of non-ionic tensides both as a surfactant and as a co-surfactant due to high stability, high performance, low toxicity, low irritancy and desirable biodegradability of many non-ionic surfactants ${ }^{34}$. However, compositional variables such as lipid content, presence of other amphiphilic entities, hydrophilic molecules or electrolytes and temperature may affect arrangement of the surfactant molecule and its efficiency to form microemulsion 35 . It has been generally observed that single chain surfactants alone do not sufficiently reduce the oil/ water interfacial tension to allow formation of a microemulsion 36. The critical micellar concentration (CMC) of high performing surfactants is smaller than those of weaker surfactants, and these more effective ones require less mass fraction to form a homogenous and stable microemulsion. The efficiency of a surfactant can be viewed from the amount of an amphiphile required to completely homogenize same quantities of oil and water ${ }^{5}$.

There is a relationship between the droplet size and amount of surfactant being employed, which implies that changes in surfactant concentration can directly affect droplet size. Increasing the surfactant concentration may result in the creation of droplets with smaller mean droplet size. This outcome could be described by the stabilization of the oil droplets because of localization of the surfactant molecules at the oil-water interface ${ }^{37}$. However, reports have shown that in some few cases the mean droplet size increase with greater surfactant concentrations 38 . This behavior could be attributed to the interfacial disruption caused by increased water penetration into the oil droplets facilitated by increased surfactant concentration, causing movement of oil droplets into the aqueous compartment.

Digestion of surfactant can change the solubilization environment of the drug which consequently cause precipitation of the poorly water soluble drugs inside the GIT ${ }^{39}$. The efficiency of self-emulsification is usually related to the Hydrophile-Lipophile Balance (HLB) value of the surfactant 40 . Surfactants are crucial components for the formulation of self-emulsifying formulations; however large amounts of surfactants cause skin irritation and wound 34 .

\section{Co-solvents/ co-surfactants}

Co-solvents like diethylene glycol monoethyl ether (Transcutol ${ }^{\circledR}$ ), propylene glycol, glycerin, polyethylene glycol (PEG) and many others, may help to solubilize large amounts of hydrophilic surfactants or the hydrophobic drugs in the lipid base. The physical state of these excipients at ambient temperature is determined by their molecular weight. PEG with molecular weight ranging from 200 to 600 is liquid at room temperature whereas those possessing molecular weight of 1000 and above, exist as thermosoftening semisolids. These solvents sometimes play the role of the co-surfactant in the microemulsions or SEDDS. The use of alcohol has some disadvantages when self-emulsifying formulation is incorporated in capsule shells causing leaching of capsule shells due to the presence of alcohol 41 . Polyethylene glycols are also known to contain varying amounts of peroxide impurities and derivatives formed by autoxidation which can activate chemical instability of the incorporated drug substances ${ }^{42}$. These impurities may chemically react with the active ingredient. These changes can be detected using technologies such as Fourier Transform Infra-Red Spectroscopy (FTIR), High Performance Liquid Chromatography (HPLC), Differential Scanning Calorimetry (DSC) and Gas Chromatography-Mass Spectroscopy (GCMS) etc

In selecting a suitable vehicle for self-emulsifying liquid preparations, drug solubility in various constituents, identification of emulsifying regions from pseudoternary phase diagram and resultant droplet size distribution need 
careful assessment and monitoring 43 . The digestibility and safety of the excipients should always be considered. Digestion of formulations will inevitably have high effect on the state of dispersion of the lipid formulation and the biofate of the drug ${ }^{44}$. Digestion of dietary triglycerides in the small intestine is very rapid and other non-ionic esters such as mixed glycerides and surfactants are also substrates of pancreatic lipase 45 .

The solubility of the drug in the oils and surfactants can be determined using the shake flask method while pseudoternary phase diagrams can be developed to identify microemulsifying regions using the water titration method. SEDDS are usually used to enhance the oral bioavailability of poorly soluble drugs (especially highly lipophilic drugs). The loaded drugs usually belong to the biopharmaceutics classification system (BCS) class II with low solubility and high permeability. SEDDS can be expressed as selfmicroemulsifying drug delivery system (SMEDDS) or selfnanoemulsifying drug delivery system (SNEDDS) depending on the size range of the oil droplet.

Therapeutic agents can have a tangible effect on the characteristics of SEDDS, such as phase characteristics and emulsion droplet size. Physical and chemical properties of the active compounds, such as log P, molecular structure and weight, presence of ionizable groups, pKa, all have significant impact on the effectiveness of SEDDS. It was observed that the addition of cefpodoxime proxetil, in selfnanoemulsifying formulations (SNEFs) reduced the nanoemulsification region in the design space when water was used ${ }^{46}$. It was observed that as $\mathrm{pH}$ of aqueous phase was changed to 1.2 , the nano-emulsification region expanded because of $\mathrm{pH}$-dependent solubility of the drug. Moreover, inclusion of bioactives into the formulations could increase the nanoemulsion droplet size relative to formulations without drug. Similar results have been reported using flurbiprofen ${ }^{47}$. The globule size of the emulsion rose with increasing concentration of drug in the SNEFs. Some drugs show surface activity and these include sodium salicylate, ascorbic acid and tricyclic amines, that may show different characteristics with an increasing concentration in formulation. Another investigation showed an enlargement of the self nanoemulsification region upon increasing the concentration of simvastatin 4-fold in the SNEFs. These reports showed that simvastatin could have moderate cosurfactant effect at the oil and water interface because of its amphiphilic nature ${ }^{48}$. Computational analysis proposed that a high number of rotatable bonds in the drug may interact with the surfactant and co-surfactant molecules. Flexibility of a molecule facilitates the formation of closely-packed stable interfacial film that forges very stable nanoemulsions.

\section{Characterization of SEDDS}

\section{(i) Visual assessment}

The self-emulsifying property of the blends and appearance of the dispersion can be understood by visual examination 49.

\section{(ii) Self-emulsification time}

The self-emulsification time is the time for a pre-concentrate SEDDS to form a homogenous, stable mixture upon dilution/ titration under specified conditions.

\section{(iii) Turbidity measurement}

This parameter reveals efficient self-emulsification by establishing if the preparation reaches equilibrium rapidly and efficiently 50 , with observation of changing turbidity as the process of self-emulsification progresses. Turbidimetric evaluation can be performed to measure the increase in turbidity until equilibrium, using a turbidimeter.

\section{(iv) Droplet size}

The droplet size distribution of SEDDS diluted with water can be determined by photon correlation spectroscopy based on the laser light scattering phenomenon. Microscopic techniques can also be used. The reduction of the droplet size to values below $50 \mu \mathrm{m}$ leads to the formation of selfmicroemulsifying drug delivery systems (SMEDDS), which are stable, isotropic and clear o/w dispersions. Polydispersity is basically calculated from the standard deviation and the mean droplet size 51, although computational techniques and advanced statistical tools are used to arrive at more accurate permutations from numerous droplets or particles with consideration of statistical errors. Polydispersity signifies the degree of uniformity of the droplet size in the formulation. The lower the value of polydispersity, then the higher the size uniformity of the formulation. The uniformity of size allows for better prediction of fate of drug based on size-dependent pharmacokinetics.

\section{(v) Zeta potential measurement}

This is used to identify the charge of the droplets dispersed in an emulsion. Most times, the charge on oil droplets in emulsion systems is negative due to presence of free fatty acids 52 .

\section{(vi) Viscosity and refractive index}

The viscosity of undiluted SEDDs is measured using viscometers or rheometers while the refractive index can be measured using a refractometer. The viscosity and refractive index are evaluated to understand the flowability and transparency respectively.

Melting properties and polymorphism of lipid or drug in self emulsifying systems may be established by differential scanning calorimetry (DSC) and X-ray diffraction.

Traditionally, preparation of SEDDS involves dissolution of drugs in oils and their mixing with suitable solubilizers.

Drugs and excipients are chemical substances with functional groups and therefore can undergo chemical reactions under suitable environmental conditions. They also undergo physical interactions. These chemical and physical changes that occur in drugs and excipients can result in the formation of new entities with different chemical, physical properties and pharmacological effects. The drug and excipient can then be said to be unstable or have undergone instability or degradation. The most frequently encountered degradative pathways are hydrolysis and oxidation-reduction reaction 53. Stability studies can be performed on pharmaceutical products to assess their thermal, physical and chemical stability. Products can be subjected to extreme temperature or stress and evaluated for physical and chemical changes. For example crystal growth and colour changes can be examined. Accelerated stability testing can also be performed to fast-track the entire process. There is a need to constantly conduct stability studies on self-emulsifying drug delivery systems.

\section{Lipid formulation classification system (LFCS)}

The LFCS briefly classifies lipid based formulations into four types according to their composition and the possible effect of dilution and digestion on their ability to prevent drug precipitation ${ }^{19}$. The classification is presented in Table 1 . 
Table 1: Lipid formulation classification system (LFCS) showing typical compositions and properties of lipid based drug delivery systems

\begin{tabular}{|l|l|l|}
\hline Formulation & Composition & Characteristics \\
\hline Type 1 & Oils without surfactant & $\begin{array}{l}\text { Non-dispersing, poor solvent capacity except for highly lipophilic drugs with } \\
\text { high solubility in oils, requires digestion to release drug. }\end{array}$ \\
\hline Type II & $\begin{array}{l}\text { Oil and water insoluble } \\
\text { surfactants. }\end{array}$ & $\begin{array}{l}\text { SEDDS, turbid o/w dispersions (particle size 0.25-2 } \mu \text { m). Unlikely to loose } \\
\text { solvent capacity to dispersion, possible loss of solvent capacity on digestion. }\end{array}$ \\
\hline Type III & $\begin{array}{l}\text { Oils - water soluble } \\
\text { surfactants and co-solvents }\end{array}$ & $\begin{array}{l}\text { SEDDS/SMEDDS, slightly bluish to clear dispersion, may lose its solvent } \\
\text { capacity upon dispersion or digestion, less easily digested }\end{array}$ \\
\hline Types IV & $\begin{array}{l}\text { Water-soluble surfactants } \\
\text { and co-solvents (oil free) }\end{array}$ & $\begin{array}{l}\text { Forms a clear micellar solution upon dispersion, likely lose solvent capacity } \\
\text { on dispersion, unlikely to be digested. }\end{array}$ \\
\hline
\end{tabular}

\section{Solid self-emulsifying drug delivery systems (solid SEDDS)}

SEDDS are usually prepared as liquid dosage forms because many excipients used in SEDDS are liquid at ambient temperature. However, due to the benefits of solid dosage forms, solid SEDDS (S-SEDDS) have been extensively investigated recently. Solid SEDDS can be presented as selfemulsifying capsules, dry emulsions, solid dispersions, tablets, beads, pellets, microspheres/nanoparticles, suppositories, implants etc. The common methods for transforming liquid and semi-solid lipid formulations into solid particles, powder or granules are spray drying, melt granulation, adsorption onto solid carriers, spray cooling, melt extrusion, super-critical fluid based methods and other established techniques 54. Equipment such as rotary pressure, two fluid or ultrasonic atomizers is used for spray cooling to atomize the liquid mixture and consequently generate droplets 55 .

Spray drying would require the atomization of the solubilized liquid formulation into a spray of droplets which are introduced into a drying chamber. The water evaporates, with the formation of dry particles under controlled temperature and air flow conditions. Solid SMEDDS has been prepared like this for the delivery of nimodipine using dextran as a solid carrier 56 .

Free flowing powders may be obtained from liquid selfemulsifying formulation by adsorption of the liquid onto solid carriers. Silica, silicates, magnesium trisilicate, talcum, magnesium hydroxide, crospovidone and cross-linked sodium carboxymethyl cellulose are typical solid carriers. Enough quantities of SEDDS can be adsorbed at high quantities (up to $70 \% \mathrm{w} / \mathrm{w}$ ) onto suitable carriers ${ }^{57}$. In melt granulation, powder agglomeration is obtained through the addition of a binder that melts or softens at relatively low temperatures. Extrusion is a production process which involves conversion of raw materials with plastic properties into a product of uniform shape and density, by forcing it through a die under controlled temperature, pressure, and flow ${ }^{58}$. The conditions or variables determine the nature of final product obtained.

A supercritical fluid-based method can also be employed either for coating of drug particles or for producing solid dispersions. The coating process entails dispersing the drug particles as powder in a supercritical fluid containing one or more dissolved coating materials. The supercritical fluid popularly used is supercritical carbon dioxide. The technique has been successfully applied for the enhancement of bioavailability of carbamazepine ${ }^{59}$.

\section{New applications of SEDDS}

Enteric-coated dry emulsion formulations have been newly developed for potential application in the oral delivery of peptide and protein drugs ${ }^{60}$. These formulations consist of a surfactant, a vegetable oil and a $\mathrm{pH}$-sensitive polymer. Oral delivery of $\beta$ - lactamase in a self-nanoemulsifying emulsion improved its bioavailability relative to the free solution 61 . Self-emulsifying tablets of non-steroidal anti-inflammatory drugs can be formulated to reduce incidents of gastro intestinal bleeding by increasing the drugs penetration through the GI mucosal membrane.

Presentation of lipid liquid formulation as a solid dosage form combines the advantages of lipid-based drug delivery systems with those of solid dosage forms. Gelled selfemulsifying formulations (SEFs) have been developed to greatly decrease the quantity of solidifying excipients required to transform SEFs into solid dosage forms, 62 . In this study, colloidal silicon dioxide (Aerosil ${ }^{\circledR} 200$ ) was applied as a gelling agent for the oily preparations. Colloidal silicon dioxide served two functions by reducing the amount of solidifying materials required and decreasing rate of drug release, thereby achieving controlled drug delivery.

In another research, SE powder formulation was prepared for the purpose of enhancing the dissolution and absorption of griseofulvin, a poorly water-soluble drug 63. Here, a significant enhancement in dissolution (without ultramicronisation) and bioavailability of griseofulvin was observed. Also a novel solid SEF of dexibuprofen was developed using spray drying technique 64 . Aerosil ${ }^{\circledR} 200$ was used as an inert solid carrier which adsorbs the liquid formulation. This allows the drug to be presented as selfemulsifying solid preparation. Solid SEDDS have been used in the delivery of diclofenac using goat fat and Tween ${ }^{\circledR} 6565$. Good drug release profile was observed with acceptable tablet properties. Also, improved oral bioavailability of halofantrine base was observed when formulated as a lipidic self-emulsifying drug delivery system ${ }^{66}$.

A new way for oral delivery of drugs with high solubility and low permeability was developed using self-double emulsifying drug delivery system (SDEDDS) ${ }^{67}$. SDEDDS can spontaneously emulsify to water-in-oil-in-water $(\mathrm{w} / \mathrm{o} / \mathrm{w})$ double emulsions in gastrointestinal fluid, with drugs entrapped in the internal water phase of the double emulsions. SDEDDS was employed to improve the oral absorption of pidotimod, a peptide like drug with high solubility and low permeability (class III of Biopharmaceutics classification system). Self-emulsifying phospholipid suspensions have been used to enhance the bioavailability and retinal accumulation of lutein ${ }^{68}$. Ability of any formulation to keep a drug in solubilized form in vivo is essential for bioavailability. In this case, rather than using excess surfactant (with its toxicity issues), high amount of phospholipid and relatively low amount of surfactant/ cosurfactant was used.

Positively charged self-emulsifying oil formulations (SEOF) have shown increased charge dependent interactions with human intestinal epithelial cells using Caco- 2 cell model ${ }^{69}$. It was suggested that positively charged SEOF caused 
increased closer adhesion of the droplets to the cell surface due to the electrostatic attraction. Many physiological studies have proved that the apical potential of absorptive cells and other cells in the body is negatively charged with respect to the mucosal solution in the lumen ${ }^{70}$. A novel nanoparticle drug delivery system consisting of chitosan and glyceryl monooleate (GMO) was developed for the effective delivery of paclitaxel. These chitosan/ GMO nanoparticles, with bioadhesive properties increased cellular association and was prepared by multiple emulsion (o/w/o) solvent evaporation methods 71 . Insulin egg yolk dispersion in selfmicroemulsifying system have been shown to suppress insulin release in $\mathrm{pH} 7.4$ phosphate buffer and protect it from enzymatic degradation in vitro in the presence of chymotrypsin 72 . A self-nanoemulsifying drug delivery system (SNEDDS) was developed for the oral delivery of zedoary turmeric oil (ZTO), an essential oil extracted from the dry rhizome of Curcuma zedoaria ${ }^{73}$. ZTO is a mixture of structurally diverse compounds which are volatile and unstable under ambient condition. In another study, solid SE sustained-release microspheres of zedoary turmeric oil (oil phase) was prepared using the quasi-emulsion-solventdiffusion method involving spherical crystallization 74 . The plasma concentration-time profiles after oral administration in rabbits showed a bioavailability of $135.6 \%$ compared with the conventional liquid SEFs. In a study, the surfactant-cosurfactant blend of Witepsol ${ }^{\circledR} \mathrm{H} 35$ and Solutol ${ }^{\circledR}$ HS15 caused enough decrease in free energy to stabilize the system ${ }^{75}$. Nanosized self-emulsifying lipid vesicles have also been used as carriers for effective delivery of lipophilic dietary supplements ${ }^{76}$. These carrier systems were proposed as alternatives to liposomes.

Oral pellets are known to overcome the poor and variable GIT absorption of drugs and have shown the capacity to minimize the influence of food on bioavailability. A new method has been developed for preparing self-emulsifying pellets by wet granulation technique using binder solution containing mono- and diglyceride oils, polysorbate 80 and the anti-inflammatory drug, nimesulide as a model active pharmaceutical ingredient 77 . In vivo studies indicated significantly higher bioavailability of the prepared pellets relative to liquid emulsion form. A comparative bioavailability study of progesterone from self-emulsifying pellet formulation, self-emulsifying solution, capsule and aqueous suspension was performed in dogs 78. A 100\% drug release was observed within a very short time for the selfemulsifying formulations. Plasma drug concentration was significantly higher when the drug was orally administered from self-emulsifying pellets and self-emulsifying solution at equal dose with others. A study demonstrated that extrusion/ spheronization technique can be used as a largescale production method for preparing solid pellets om the liquid precursors to improve oral absorption and bioavailability ${ }^{79}$. SE pellets of nitrendipine, a hydrophobic drug, were prepared using this technique. Another study showed that the release of methyl hydroxybenzoate (methylparaben) and propyl hydroxybenzoate (propylparaben) from pellet formulations could be controlled by incorporating them into self-emulsifying systems containing water-soluble plasticizer and talc 80 .

An isotropic formulation of loratadine consisting of Captex ${ }^{\circledR}$ 200, Cremophore ${ }^{\circledR}$ EL and Capmul $^{\circledR}$ MCM have been prepared as Self-emulsifying beads 81 . The SE mixture was loaded onto polypropylene beads (PPB) using the solvent evaporation method. Formulations were optimized for loading efficiency and in vitro drug release.

An in vitro release study showed that a prolonged release self-emulsifying PLGA wafers of carmustine released entrapped drug for 7 days which explains the observed higher in vitro anti-tumor activity 82 . Another research showed the formulation of self-emulsifying solid lipid microparticles as viable systems for delivery of glibenclamide 83 .

An interesting technique for improving the oral bioavailability of poorly soluble drugs is the use of the concept of supersaturation for design of supersaturable formulations. Supersaturable SEDDS formulations have been designed and developed to reduce the surfactant side effects and achieve rapid absorption of poorly soluble drugs ${ }^{84}$. The supersaturable SEDDS formulations contain a lower level of surfactant and also contain polymeric precipitation inhibitor to yield and stabilize a drug in a temporarily supersaturated state. Hydroxypropyl methylcellulose (HPMC) and related cellulose polymers are known to inhibit crystallization and thereby create the supersaturated state 85 . Supersaturation allows activity of drug beyond its solubility limit and facilitates drug passage across the biological barrier 86 .

In terms of future outlook, as scientists continue to discover new drug moieties with poor aqueous solubility, there would always be the need for an appropriate drug delivery system. Highly lipophilic drugs are always candidates for SEDDS. Furthermore, advanced research in the field of biotechnology has produced numerous bioactive protein and peptide molecules which require special formulation techniques. Self-emulsifying drug delivery systems are gradually showing some promise as a platform for effective oral delivery of these biological substances. SEDDS would also be useful in the delivery of phytopharmaceuticals. Recently many plant derived bioactives including volatile oils, have been formulated as self-emulsifying lipid formulations.

Many pharmaceutical products based on self-emulsifying drug delivery systems have been marketed.

\section{Conclusion}

Self-emulsifying formulations (SEFs) allow oil-soluble bioactive entities to be effectively dispersed in a lipid and solubilized for improved dissolution, absorption and bioavailability. Upon dispersion in GIT fluid, these formulations self-emulsify, thereby generating fine droplets of disperse phase. As more chemical moieties and lead compounds are discovered with poor aqueous solubility, SEDDS or SEFs are increasingly becoming attractive as delivery systems. Also ethnomedicines are promising candidates to be presented using SEDDS. More research need to be performed to investigate drug targeting using these systems.

\section{References}

1. Gursoy RN, Benita S. Self-emulsifying drug delivery systems (SEDDS) for improved oral delivery of lipophilic drugs. Biomed Pharmacother 2004; 58:173-182.

2. Wakerly MG, Pouton CW, Meakin BJ. Evaluation of the self emulsifying performance of a non ionic surfactant-vegetable oil mixture. J Pharm Pharmacol 1987; 39:6.

3. Fahy E, Subramaniam S, Murphy R, Nishijima M, Raect C, Shimizu T, Spener F, Van Meer G, Wakelam M, Dennis EA. Update of the LIPID MAPS comprehensive classification system for lipids. J Lipid Res 2009; 50 (supplement):59-514.

4. Subramaniam S, Fahy E, Gupta S, Sud M, Byrnes RW, Cotter D, Dinasarapu AR, Maurya MR. Bioinformatics and systems biology of the lipidome. Chem Rev 2011; 111 (10):6452-6490.

5. Djekic L, Primorac M. The Influence of cosurfactants and oils on the formation of pharmaceutical microemulsions based on PEG8 caprylic/capric glycerides. Int J Pharm 2008; 352:231-239. 
6. Tsevegsuren N, Aitzetmuller K, Bruhl L, Werner G. Seed oil fatty acids of Mongolian compositae: The trans fatty acids of Heteropappus hispidus, Asterothamnus centrali-asiaticus and Artemisia palustris. J High Resol Chromatogr 2000; 23:360-366.

7. Bell SJ, Bradley D, Forse RA, Bistrian BR. The new dietary fats in health and disease. J Am Diet Assoc 1997; 97:280-286

8. Schneider M. Industrial production of phospholipids - lecithin processing. Lipid Technology 1997:109-116.

9. Stuchlik M, Zaks S. Lipid-based vehicle for oral drug delivery Biomed Papers 2001; 154(2):17-26.

10. Mistry RB, Sheth NS. A Review: Self emulsifying drug delivery system. International Journal of Pharmacy and Pharmaceutical Sciences 2011; 3 (2):23-28.

11. Constantinides PP. Lipid microemulsions for improving drug dissolution and Oral absorption: Physical and biopharmaceutical aspects. Pharm. Res 1995; 12:1561-1572.

12. Groves MJ, Mustata RMA. Measurement of spontaneity of selfemulsifiable oils. J Pharm Pharmacol 1974; 26:671-688.

13. Jannin V, Musakhanian J, Marchaud D. Approaches for the development of solid and semi solid lipid based formulations. Adv Drug Deliv Rev 2007; 60:734-746.

14. Serajuddin A.T.M. Solid dispersion of poorly watersoluble drugs: early promises, subsequent problems, and recent breakthroughs. J. Pharm. Sci. 1999; 88:1058-1066

15. Helfrich W. Elastic properties of lipid bilayers: Theory and possible experiments. Z. Naturforsch, 1973; 28c:693-703

16. Bancroft W.D. The theory of emulsification V. Journal of Physical Chemistry 1913; 17:501-519

17 Israelachvili JN. Theory of self-assembly of hydrocarbon amphiphiles into micelles and bilayers. Journal of the Chemical Society, Faraday Transactions 2: Molecular and Chemical Physics 72:1525-1568

18. Agubata C.O, Nzekwe I.T, Obitte N.C, Ugwu C.E, Attama A.A Onunkwo G.C. Effect of oil, surfactant and co-surfactant concentrations on the phase behavior, physicochemical properties and drug release from self-emulsifying drug delivery systems. Journal of Drug discovery, Development and Delivery 2014; 1:1-7.

19. Pouton CW. Formation of self-emulsifying drug delivery System. Adv Drug Deliv Rev 1997; 25:47-58.

20. El Laithy HM. Self-nanoemulsifying drug delivery system for enhanced bioavailability and improved hepatoprotective activity of biphenyl dimethyl dicarboxylate. Curr. Drug Del 2008; 5:170176.

21. Benet L. The drug efflux- metabolism alliance biochemical aspects. Adv Drug Deliv Rev 2001; 50:S3-S11.

22. Devani M, Ashford M, Craig DQ. The emulsification and solubilisation properties of polyglycolysed oils in selfemulsifying formulations. J Pharm Pharmacol 2004; 56:307-316.

23. Huass DJ, Fogal SE, Ficorilli JV, Price CA, Roy T, Jayaraj AA, Keirns JJ. Lipid based delivery systems for improving the bioavailability and lymphatic transport of poorly water soluble $\mathrm{LTB}_{4}$ inhibitor. J Pharm Sci 1998; 87(2):164-169.

24. Caliph SM, Charman WN, Porter CJ. Effect of short-, medium- and long chain fatty acid-based vehicles on the absolute oral bioavailability and intestinal lymphatic transport of halofantrine and assessment of mass balance in lymph cannulated and non cannulated rats. J Pharm Sci 2000; 89:1073-1084.

25. Charman WN, Stella VJ. Transport of lipophilic molecules by the intestinal lymphatic system. Adv Drug Deliv Rev 1991; 7:1-14.

26. Pouton CW. Lipid formulations for oral administration of drugs. Non-emulsifying, self emulsifying and self microemulsifying drug delivery systems. Eur J Pharm Sci 2000; 11:S93-98.

27. Tang JL, Sun J, and He ZG. Self-emulsifying drug delivery systems: strategy for improving oral delivery of poorly soluble drugs. Current Drug Therapy 2007; 2 (1):85-93.

28. Dahan A, Hoffman A. Rationalising the selection of oral lipid based drug delivery systems by an in vitro dynamic lipolysis model for improved oral bioavailability of poorly water soluble drugs. J Control Rel 2008; 129:1-10.

29. Antron N, Vandamme TF. The universality of low energy nanoemulsification. Int J Pharm 2009; 377:142-147.

30. Aungst BJ. Intestinal permeation enhancers. J Pharm Sci 2000; 89:429-442.

31. Talegaonkar S, Azeem A, Ahmad FJ, Khar RK, Pathan SA, Khan ZI. Microemulsion: A novel approach to enhanced drug delivery. 238 Recent Patents on Drug Delivery and Formulation 2008; 2:238-257.

32. Bagwe RP, Kanicky JR, Palla BJ, Patanjali PK, Shah DO. Improved drug delivery using microemulsions: rationale, recent progress, and new horizons. Critical Reviews in Therapeutic Drug Carrier Systems 2001; 18 (1): 77-140.

33. Meinzer A, Mueller E, Vonderscher J. Microemulsion- A suitable galenical approach for the absorption enhancement of a low soluble compound? BT Gattefosse 1995; 88:21-26.

34. Lawrence MJ, Rees GD. Microemulsion-based media as novel drug delivery system. Adv Drug Deliv Rev 2000; 45:89-121.

35. Kahlweit M. Microemulsions. Annu. Rep. Prog. Chem-sect. C. 1999; 95:89-115.

36. Bhargava HN, Narurkar A, Lieb LM. Using microemulsions for drug delivery. Pharm Tech 1987; 11:46-52.

37. Karim A, Gokhale R, Cole M, Sherman J, Yeramian P, Bryant M, Franke H. HIV protease inhibitor SC-52151: a novel method of optimizing bioavailability profile via a microemulsion drug delivery system. Pharm Res 1994; 11:S368.

38. Georgakopoulos E, Farah N, Vergnault G. Oral anhydrous nonionic microemulsions administered in softgel capsules. B T Gattefosse 1992; 85:11-20.

39. Cui J, Yu B, Zhao Y, Zhu W, Li H, Lou H, Zhai G. Enhancement of oral absorption of curcumin by self microemulsifying drug delivery systems. Int J Pharm 2009; 371:148-155.

40. Thi TD, Van Speybroeck M, Barillaro V, Martens J, Annaert P, Augustijns P, Van Humbeeck J, Vermant J, Van den Mooter G. Formulate-ability of ten compounds with different physicochemical profiles in SMEDDS. Eur J Pharm Sci 2009; 38: 479-488.

41. Patel PA, Chaulang GM, Akolkotkar A, Mutha SS, Hardikar SR, Bhosale AV. Self emulsifying drug delivery systems: A review. Research J. Pharm and Tech. 2008; 1(4):313-323.

42. Porter CJ, Charman WN. In vitro assessment of oral lipid based formulations. Adv Drug Deliv Rev 2001; 50 suppl 1: S127-47.

43. Komuru TR, Gurley B, Khan MA, Reddy IK. Self-emulsifying drug delivery systems (SEDDS) of Co enzyme Q 10: formulation development and bioavailability assessment. Int J Pharm 2001; 212:233-246.

44. MacGregor KJ, Embleton JK, Lacy JE, Perry EA, Solomon LJ, Seager $\mathrm{H}$, Pouton CW. Influence of lipolysis on drug absorption from the gastrointestinal tract. Adv Drug Deliv Rev 1997; 25:33-46.

45. Embleton JK, Pouton CW. Structure and activity of gastrointestinal lipases. Adv Drug Deliv Rev 1997; 25:15-32.

46. Date AA, Nagarsenker MS. Design and evaluation of self nanoemulsifying drug delivery system (SNEDDS) for cefpodoxime proxetil. Int J Pharm 2007; 329:166-172.

47. Wang L, Dong J, Eastoc J, LI X. Design and optimization of a new self-nanoemulsifying drug delivery system. J Colloid Interface Sci 2009; 330:443-448.

48. Dixit RP, Nagarsenker MS. Formulation and in vivo evaluation of self-nano emulsifying granules for oral drug delivery of a combination of ezetimibe and simvastatin. Drug Dev Ind Pharm 2008; 34:1285-1296.

49. Shah NH, Carvajal MT, Patel CJ, Infeld MH, Malick AW. Self emulsifying drug delivery systems (SEDDS) with polyglycolized glycerides for improving in vitro dissolution and oral absorption of lipophilic drugs. Int J Pharm 1994; 106:15-23.

50. Lievens HSR, Craig DQM, Pitt KG, Storey DE. An investigation into physico-chemical properties of self-emulsifying systems using 
low frequency dielectric spectroscopy, surface tension measurement and particle size analysis. Int J Pharm 1993; 96:147-55

51. Baboota S, Shakeel F, Ahuja A, Ali J, Shafiq S. Design, development and evaluation of novel nanoemulsion formulations for transdermal potential of celecoxib. Acta Pharm. 2007; 57:315332.

52. Maurya SD, Arya RKK, Rajpal G, Dhakar RC, Self-micro emulsifying drug delivery systems (SMEDDS): a review on physico-chemical and biopharmaceutical aspects, Journal of Drug Delivery and Therapeutics 2017; 7(3):55-65

53. Lachman L, Lieberman, HA. The theory and practice of industrial pharmacy, CBS Publishers, New Delhi, special Indian edition, 2009; p 772.

54. Suman K, VSR Chandrasekhar P, Balaji S. Approaches for the development of solid self emulsifying drug delivery systems and dosage forms. Asian J Pharm Sci 2009; 4 (4): 240-253.

55. Rodriguez L, Passerini N, Cavallari C, Albertini B, Fini A. Description and preliminary evaluation of a new ultrasonic atomizer for spray-congealing processes. Int J Pharm 1999; 183 (2):133-143.

56. Yi T, Wan JL, Xu HB, Yang X. A new solid self microemulsifying formulation prepared by spray-drying to improve the oral bioavailability of poorly water soluble drugs. Eur J Pharm Biopharm 2008; 70:439-444.

57. Ito Y, Kusawake T, Ishida M, Tawa R, Shibata N, Takada K. Oral solid gentamicin preparation using emulsifier and adsorbent. J Control Rel 2005; 105 (1-2):23-31.

58. Verreck G, Brewster ME. Melt extrusion based dosage forms: Excipients and processing conditions for pharmaceutical formulations. Bull Tech Gattefosse 2004; 97:85-95.

59. Ribeiro Dos Santos I, Thies C, Richard J, Le Meurlay D, Gajan V, Vandevelde V, Benoit JP. A supercritical fluid based coating technology 2: solubility considerations. J Microencapsul 2003; 20 (1):97-109.

60. Toorisaka E, Haashida M, Kamiya N, Ono H, Kokazu Y, Goto M. An enteric coated dry emulsion formulation for insulin delivery. J Control Rel 2005; 107:91-96.

61. Rao SV, Agarwal P, Shao J. Self nanoemulsifying drug delivery systems (SNEDDS) for oral delivery of protein drugs: II. In vitro transport study. Int J Pharm 2008; 362:10-15.

62. Patil P. Effect of formulation variables on preparation and evaluation of gelled self-emulsifying drug delivery system (SEDDS) of ketoprofen. AAPS Pharm Sci Tech 2004; 5:34-42.

63. Arida AI, Moawia MA, Hantash AJ, Moawia MA, Hantash AJH. Improving the high variable bioavailability of griseofulvin by SEFs. Chem Pharm Bull 2007; 55:1713-1719.

64. Balakrishnan P, Lee BJ, Lee YI, Woo JS, Yong CS, Choi HG. Enhanced oral bioavailability of coenzyme Q10 by selfemulsifying drug delivery systems. Int J Pharm 2009; 374:66-72.

65. Attama AA, Nzekwe IT, Nnamani PO, Adikwu MU, Onugu CO. The use of solid self- emulsifying system in the delivery of diclofenac. Int J Pharm 2003; 262:23-28.

66. Khoo S, Humberston AJ, Porter CJH, Edwards GA, Charman WN. Formulation, design and bioavailability assessment of lipidic self-emulsifying formulations of halofantrine. Int J Pharm 1998; 167:155-164.

67. Qi X, Wang L, Zhu J, Hu Z, Zhang J. Self-double-emulsifying drug delivery system (SDEDDS): A new way for oral delivery of drugs with high solubility and low permeability. Int J Pharm 2011; 409:245-251.

68. Shanmugam S, Park J, Kim KS, Piao ZZ, Youg CS, Choi H, Woo JS. Enhanced bioavailability and retinal accumulation of lutein from self-emulsifying phospholipid suspension (SEPS). Int J Pharm 2001; 412:99-105.

69. Gershanik T, Haltner E, Lehr CM, Benita S. Charge dependent interaction of self-emulsifying oil formulations with Caco-2 cells nanolayers; binding effects on barrier function and cytotoxicity. Int J Pharm 2000; 211:29-36.

70. Corbo DC, Liu JC, Chien YW. Characterization of the barrier properties of mucosal membranes. J Pharm Sci 1990; 79:202206.

71. Trickler WJ, Nagvekar AA, Dash AK . A novel nanoparticle formulation for sustained paclitaxel delivery. AAPS Pharm Sci Tech 2008; 9 (2):486-493.

72. Singnurkar PS, Gidwani SK. Insulin- egg yolk dispersions in selfmicroemulsifying system. Indian J Pharm Sci 2008; 70(6):727732.

73. Zhaoa Y, Wanga C, Chowh AHL, Renc K, Gongc T, Zhangc Z, Zhenga Y. Self-nanoemulsifying drug delivery system (SNEDDS) for oral delivery of zedoary essential oil: formulation and bioavailability studies. Int J Pharm 2010; 383:170-177.

74. You, J. Study of the preparation of sustained-release microspheres containing zedoary turmeric oil by the emulsionsolvent-diffusion method and evaluation of the selfemulsification and bioavailability of the oil. Colloids Surf. B Biointerfaces 2006; 48:35-41.

75. Nepal PR, Han HK, Choi HK. Preparation and in vitro-in vivo evaluation of Witepsol ${ }^{\circledR} \mathrm{H} 35$ based self-nanoemulsifying drug delivery systems (SNEDDS) of coenzyme Q10. Eur J Pharm Sci 2010; 39:224-232.

76. Koynova R, Mariana T. Nanosized self-emulsifying lipid vesicles of diacylglycerol-PEG lipid conjugates: Biophysical characterization and inclusion of lipophilic dietary supplements. biochim biophys acta 2010; 1798:646-653.

77. Franceschinis E, Voinovich D, Grassi M, Perissutti Filipovic-grcic BJ, Martinac A, Meriani-merlo F. Self-emulsifying pellets prepared by wet granulation in high-shear mixer: influence of formulation variables and preliminary study on the in vitro absorption. Int J Pharm 2005; 291:87-97.

78. Tuleu C. Comparative bioavailability study in dogs of a selfemulsifying formulation of progesterone presented in a pellet and liquid form compared with an aqueous suspension of progesterone. J Pharm Sci 2004; 93: 1495-1502.

79. Wang Z, Sun J, Wang Y, Liu X, Liu Y, Fu Q, Meng P, He Z. Soli d selfemulsifying nitrendipine pellets: preparation and in vitro/in vivo evaluation. Int J Pharm 2010; 383:1-6.

80. Serratoni M, Newton M. Controlled drug release from pellets containing water-insoluble drugs dissolved in a self-emulsifying system. Eur J Pharm Biopharm 2007; 65:94-98.

81. Patil P, Paradkar A. Porous polystyrene beads as carriers for selfemulsifying system containing loratadine. AAPS Pharm Sci Tech 2006; 7:E1-E7.

82. Chae GS, Lee JS, Kim SH. Enhancement of the stability of BCNU using self-emulsifying drug delivery systems (SEDDS) and in vitro antitumor activity of self-emulsified BCNU-loaded PLGA wafer. Int J Pharm 2005; 301:6-14.

83. Agubata CO, Onwumere OC, Oraeluno JN, Obasi JC, Chidebelu CC, Akpa PA. Self-emulsifying solid lipid microparticles of glibenclamide based on amphiphilic non-ionic caprylocaproyl macrogol-8-glyceride. International Journal of Modern Pharmaceutical Research 2019; 3(6):22-30

84. Poelma FG, Breas R, Tukker JJ, Crommelin DJ. Intestinal absorption of drugs. The influence of mixed micelles on the disappearance kinetics of drugs from the small intestine of the rat. J Pharm Pharmacol 1991; 43:317-324.

85. Pellet MA, Davis AF, Hadgraft J. Effect of supersaturation on membrane transport: 2. piroxicam. Int J Pharm 1994; 111:1-6.

86. Gao P, Guyton ME, Huang T, Bauer JM, Stefanski KJ, Lu Q. Enhanced oral bioavailability of a poorly water soluble drug PNU-91325 by supersaturable formulations. Drug Dev Ind Pharm 2004; 30:221-229. 